Journal de la Société des Océanistes

\section{Journal de la Société des Océanistes}

140 | janvier-juin 2015

Intégration régionale des territoires français dans le Pacifique Sud

\title{
Horizons régionaux et variations océaniennes des territoires français
}

Regional Perspectives and Oceanian Variations for the French Territorie

Rudy Bessard et Nathalie Mrgudovic

\section{(2) OpenEdition}

Journals

Édition électronique

URL : http://journals.openedition.org/jso/7224

DOI : $10.4000 /$ jso. 7224

ISSN : $1760-7256$

Éditeur

Société des océanistes

Édition imprimée

Date de publication : 15 juin 2015

Pagination : 5-20

ISBN : 9782854301250

ISSN : 0300-953x

Référence électronique

Rudy Bessard et Nathalie Mrgudovic, « Horizons régionaux et variations océaniennes des territoires

français », Journal de la Société des Océanistes [En ligne], 140 | janvier-juin 2015, mis en ligne le 05 juillet 2015, consulté le 23 septembre 2020. URL : http://journals.openedition.org/jso/7224 ; DOI : https://doi.org/10.4000/jso.7224 


\title{
Horizons régionaux et variations océaniennes des territoires français
}

\author{
par \\ Rudy BESSARD* et Nathalie MRGUDOVIC**
}

"Liidentité régionale [...] repose sur une réalité concrète: la mer est aussi réelle que vous et moi [...]. Je ne fais que constater qu'elle forge le caractère de cette planète, qu'elle est une source majeure de notre subsistance et que nous l'avons tous en partage, où que nous soyons en Océanie. Mais au-delà des épreuves quotidiennes, la mer nous lie les uns aux autres. Elle est source de sagesse infinie. La mer est notre métaphore la plus puissante. L'océan est en nous. » (Hau’Ofa, 2015 [1997] : 55-56)

Souvent présentés de manière séparée, les territoires français dans le Pacifique Sud, objets de ce dossier, possèdent cependant des horizons régionaux intéressants qui mettent en exergue la variété des écosystèmes politiques de l'espace océanien ${ }^{1}$. Les facettes multidimensionnelles des évolutions humaines montrent une " mer d'îles " océaniennes en mouvement, selon l'intellectuel tongien Epeli Hau’Ofa (2008 [1993]). Sa pensée alimente la formation d'une identité régionale océanienne, à partir du socle d'un projet commun d'unir les peuples du Pacifique autour de la protection du Pacifique Sud, dont les habitants seraient les "gardiens de l'océan" (Hau'Ofa, 2015 [1997]). La formation d'une identité régionale ne masque cependant pas la diversité des sociétés océaniennes qui évoluent au gré de trajectoires historiques et culturelles variées, en étant de plus en plus irriguées par des flux d'horizons internationaux et régionaux. Ainsi, les dynamiques de ces sociétés montrent qu'elles ne sont pas dominées malgré elles par un subconscient précolonial (Doumenge, 2001 ; Wit- tersheim, 2014), ni figées par le legs colonial ou prisonnières d'une réification des cultures dans le sillage de la modernité et de la mondialisation (Wolton, 2013 ; Babadzan, 1999, 2009).

Peut-on pour autant affirmer que les trois principaux territoires français du Pacifique Nouvelle-Calédonie, Polynésie française, Wallis-et-Futuna - se retrouvent dorénavant en situation postcoloniale? Si les deux premiers de ces trois territoires sont inscrits sur la liste des Nations unies des territoires non autonomes à décoloniser (Regnault, 2013), ils ont connu une évolution institutionnelle importante, entre la "décolonisation dans la République" (Mohamed-Gaillard, 2010) mais sans l'indépendance et l'approche de la décolonisation au sens du droit international qui est privilégiée par les indépendantistes et dont les étapes transitoires ne concordent pas nécessairement avec les possibilités du droit français (Leblic, 1993; Regnault, 2013 ; Faberon, 2008 ; Graff, 2012 ; voir aussi le débat entre Alain Christnacht, conseiller d'État, et Roch Wamytan, leader indépendantiste kanak [Regnault et Fayaud, 2008 : 108-110]). Deux volumes du JSO ont été consacrés à ces deux territoires français, le premier coordonné par Isabelle Leblic, à l'occasion du cent cinquantenaire de la colonisation en Nouvelle-Calédonie (117 de 2003) et le second sur la Polynésie française (119 de 2004). Quant au territoire de Wallis-etFutuna, il demeure régi par la loi du 29 juillet 1961 qui a transformé cet ancien protectorat français en Territoire d'Outre-Mer (том) (voir le

\footnotetext{
* Chercheur associé en Science politique, Universités de Bordeaux (Centre Montesquieu de recherches politiques) et de Polynésie française (Laboratoire Gouvernance et développement insulaire), rbessard7@gmail.com.

** Maître de conférences, Aston University (Grande-Bretagne), associée au Laboratoire Gouvernance et développement insulaire,n.mrgudovic@aston.ac.uk.

1. Plusieurs articles de ce dossier sont tirés des interventions présentées au $2^{\mathrm{e}}$ colloque e-toile Pacifique à l'EHESs Paris (3-5 octobre 2013), dans la session du intitulée "L'intégration régionale autour des trois entités françaises »
} 


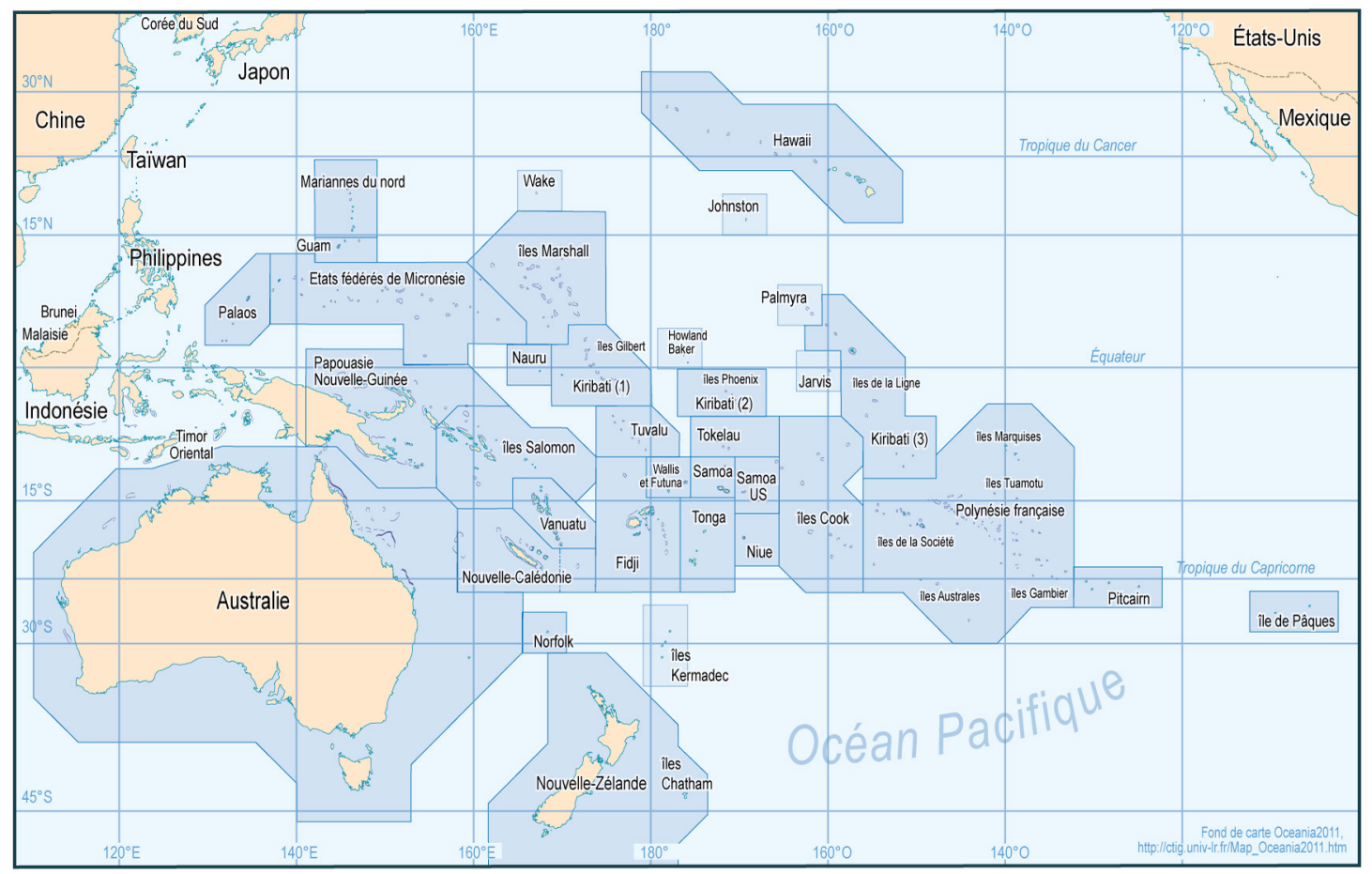

CARTE 1. - L'Océanie (http://ctig.univ-lr.fr/Fond_carte/Fond_Oceania2011_Col_Big.png)

dossier Spécial Wallis-et-Futuna du JSO 122-123 coordonné par Hélène Guiot et Isabelle Leblic en 2006).

Le courant pluridisciplinaire du post-colonialisme (anthropologues, sociologues, historiens, politologues, écrivains), apparu en France dans le sillage de Georges Balandier, s'interroge sur le rapport à l'histoire des peuples colonisés, ainsi que sur l'hybridité qui résulte de ces situations coloniales diverses, illustrant notamment la permanence des conséquences de la colonisation, aussi bien dans les sociétés anciennement colonisées qu'au sein des sociétés colonisatrices, qui ressentent ensemble un "besoin d'histoire " (Smouts, 2007). Sans s'appesantir sur le bienfondé des postcolonial studies, quelquefois marquées par un parti-pris et une radicalité qui freinent leur implantation (Smouts, 2007), ces études ont pour mérite de présenter différents points de vue, en prenant en compte les degrés du traumatisme colonial et l'hybridité des pratiques sociales et culturelles à l'œuvre dans ces territoires d'outre-mer. Ainsi, la situation contemporaine des territoires français du Pacifique connaît d'intéressantes dynamiques sociales, politiques et économiques, que les chercheurs s'efforcent de mettre en lumière, en s'appuyant sur une interdisciplinarité nécessaire à l'étude de ces terrains de recherche. Marqués par la complexité, ils amènent à réfuter les préjugés coloniaux, raciaux ou une approche trop occidentalo-centrée sur des réalités relevant d'univers cosmogoniques, sociaux et culturels différents, conduisant au fait que «la recherche d'universaux s'en trouve déplacée " (Monnerie, 2014 : 150). Avec les dynamiques régionales de l'espace océanien ( $c f$. infra), les "dynamiques du dehors » ont longtemps été étudiées dans le champ des relations internationales par un regard extérieur axé sur les intérêts des grandes puissances, en délaissant les perceptions des acteurs océaniens (Mohamed-Gaillard, 2012). La négligence de l'attention portée sur les " dynamiques du dedans » a été en partie contrebalancée dès les années 1980 par l'approche "Islander-oriented" et la prise en considération des réflexions océaniennes (Leblic, 1993, 2003 ; Lal, 2007). Cependant, la lecture océanienne des enjeux internationaux demeure marginale, aussi bien sur la scène internationale que dans les Pacific Studies (Mohamed-Gaillard, 2012).

Pourtant, l'analyse historique des relations internationales peut être effectuée à la lumière des jeux d'échelles entre situations particulières et contextes généraux, ainsi que le démontre l'historienne Sarah Mohamed-Gaillard, dans le précédent dossier du Journal de la Société des Océanistes (JSO) publié sous sa direction. Le dossier Relations internationales et régionales en Océanie, publié dans numéro 135, faisait le bilan des publications de la revue dans le domaine des relations internationales relatives à l'Océanie. Plusieurs dossiers et de nombreux articles sur ces thématiques ont donc déjà été publiés par le $J S O$, depuis le volume 87 traitant de l'Océanie et de ses problèmes géostratégiques (1988), le numéro double 92-93 relatif à la Pacific Way et aux rela- 
tions régionales (1991), le numéro traitant des défis du Pacifique (JSO 125, 2007), puis de la question de l'environnement (JSO 126-127, dirigé par Jean Trichet et Isabelle Leblic, voir Trichet et Leblic, 2008 : 5-10), ou les articles de Stephanie Lawson (JSO 109, 1999), Nathalie Mrgudovic (JSO 117, 2003), Guy Agniel (JSO 126-127, 2008 : 145-151) ou Stéphanie Graff (JSO 134, $2012: 61-83)$ pour ne citer que les plus récents. Si le dossier du volume 135 du JSO montrait la vulnérabilité stratégique puis économique des territoires insulaires océaniens dans la globalisation, faisant de l'Océanie un continent en marge des enjeux internationaux, il mettait aussi en évidence la consolidation des puissances régionales australasiennes, l'intérêt croissant des puissances asiatiques dans la région (Mohamed-Gaillard, 2012 : 180), ainsi que les effets des politiques d'aide au développement (Overton et al., 2012). De manière plus épisodique, quelques revues se sont penchées sur la dimension internationale de la question océanienne. Par exemple, la revue Hermès (Barbe et Meltz, 2013) a consacré un dossier au "monde Pacifique dans la mondialisation ", faisant appel à divers spécialistes des champs relatifs à la linguistique, aux techniques, à l'art, aux patrimoines et aux cultures en Océanie. Mais la démarche scientifique visant à étudier le champ des relations internationales dans la région océanienne s'inscrit dans la continuité des publications du Journal de la Société des Océanistes sur ce thème. En particulier, dans le sillage du volume 135 , le présent dossier ouvre une nouvelle entrée dans l'étude des relations océaniennes en mettant l'accent sur la question de l'intégration régionale autour des territoires français dans le Pacifique. En l'occurrence, ce dossier s'appuie sur une perspective davantage géopolitique, institutionnelle qu'une étude de la dimension culturelle, sociologique et anthropologique de la région, qui aurait évidemment complété son contenu. Les travaux scientifiques déjà publiés en la matière, en particulier par le Journal de la Société des Océanistes, sont éclairants sur la profondeur ethnologique des sociétés océaniennes.

Les articles de ce dossier sont consacrés plus spécifiquement aux territoires français et à une problématique d'intégration régionale, essentiellement dans les champs politique et institutionnel. Il s'agit de mettre en évidence certaines dynamiques du processus de régionalisation dans le Pacifique et d'observer plus précisément ce que recouvre en réalité un concept en apparence aussi flou que celui d'intégration régionale. D'autant plus qu'une interrogation sur les possibilités et les facteurs d'inté- gration prend en compte la question " qui intègre qui ? », en raisonnant à partir du jeu des acteurs (dirigeants politiques et acteurs sociaux), mais aussi en fonction du cadre posé par les institutions (États, exécutifs locaux, organisations régionales et internationales), ainsi que par les différentes formes de leadership qui en émanent. En particulier, la formulation de la politique de la France par son représentant dans le Pacifique, M. Christian Lechervy, propose une réflexion intéressante qui met en relief les nouvelles problématiques régionales autour de l'insertion des territoires français dans les institutions de l'espace océanien. En resituant les enjeux à partir des spécificités de ces territoires, il apparaît que les formes institutionnelles de l'intégration régionale dans le Pacifique Sud ne possèdent pas le même caractère de rétrocession de la souveraineté, qui n'est d'ailleurs pas toujours juridiquement pleine et entière (Kochenov, 2011). Cependant, la formation d'une identité régionale, décelable par exemple dans les lieux d'échanges culturels (Jeux du Pacifique, Festival des Arts du Pacifique), irrigue les canaux vecteurs d'intégration régionale à plusieurs égards, par delà le champ culturel. De la sorte, la notion d'intégration régionale peut être envisagée au sens large de la rencontre, de l'échange et de la coopération entre deux ou plusieurs territoires/ États et types d'acteur de ces territoires (politiques, économiques, culturels, religieux, etc.).

\section{Trois territoires aux caractéristiques spatiales et socioéconomiques différentes}

Les trois principaux territoires français ${ }^{3} \mathrm{du}$ Pacifique - Nouvelle-Calédonie, Polynésie française, Wallis-et-Futuna - possèdent des caractéristiques soulignant leurs différences de position géographique, de population, de superficie, de statut ou de leurs langues vernaculaires (Vernaudon, 2010), mais ils n'en sont pas pour autant dépourvus de ramifications.

En-dehors d'un statut institutionnel propre à chacun des territoires - trois royaumes dans la Collectivité d'Outre-Mer (сом) de Walliset-Futuna cohabitant avec un administrateur supérieur, statut d'autonomie sui generis issu de l'accord de Nouméa (1998) pour la NouvelleCalédonie, statut d'autonomie pour la сом de Polynésie française (2004, révisé en 2007) -, leurs caractéristiques sociales et économiques fluctuent également. Ainsi, la Nouvelle-Calédonie possède une économie dynamique, contrairement aux deux autres territoires. Axée sur le

2. Une question qui a été notamment posée par le sociologue Bruno Saura au sujet du débat sur le métissage et sa conceptualisation en Polynésie française (Saura, 2008).

3. Voir les trois JSO mentionnés qui leur ont déjà été consacrés. 
secteur minier (voir le $\mathrm{n}^{\circ} 138-139$ du JSO: Les mises en récit de la mine dans le Pacifique) par ses gisements importants de nickel $(90 \%$ des exportations d'où l'importance du cours fluctuant du minerai), l'économie calédonienne s'appuie aussi sur la pêche, l'aquaculture ou l'agriculture (élevage). Avec une superficie de $18575 \mathrm{~km}^{2}$, le territoire est réparti entre la Grande Terre $\left(16361 \mathrm{~km}^{2}\right)$, les îles Loyauté, Belep, l'île des Pins, auxquelles s'ajoutent de nombreux îlots et récifs, qui portent la zone économique exclusive à $1422000 \mathrm{~km}^{2}$, soit $13 \%$ de la zeE française. Marquée par une histoire singulière qui a entraîné une répartition de la population entre le peuple autochtone kanak (40,3\% en 2009), les Européens (29,2 \%), Wallisiens et Futuniens $(8,7 \%)$, Tahitiens (2\%), Indonésiens (1,6\%), Vietnamiens $(1 \%)$ ou Ni-Vanuatu $(0,8 \%)$ et autres, la population résidant en Nouvelle-Calédonie s'élevait à 268767 habitants en 2014, dont les deux tiers (179 509 habitants) dans et autour de la ville de Nouméa (Institut de la statistique et des études économiques de NouvelleCalédonie - ISEE).

Le territoire de Wallis-et-Futuna est composé de trois îles principales - l'île volcanique basse de Wallis, entourée d'un lagon, est séparée par $230 \mathrm{~km}$ des îles volcaniques hautes de Futuna et Alofi (inhabitée), dépourvues de lagon -, Wallis-et-Futuna possède une superficie de $142 \mathrm{~km}^{2}$ pour une zEE de $300000 \mathrm{~km}^{2}$. Le nombre d'habitants était de 12197 habitants en 2013, dont les deux tiers se trouvent à Wallis (Service territorial de la statistique et des études économiques de Wallis-et-Futuna - STSEE). La baisse de l'accroissement naturel (perte de 2750 habitants depuis 2003 soit $18 \%$ de la population essentiellement polynésienne) est principalement due à un courant migratoire en direction du pôle d'attractivité de la Nouvelle-Calédonie voisine (voir infra) : 21262 personnes se déclarant wallisiennes et/ ou futuniennes étaient recensées en NouvelleCalédonie en 2009, soit presque le double de la population résidant au Fenua ("pays»). L'économie du territoire est basée sur les dotations de l'État (environ $80 \%$ du budget) et le secteur public, qui représente $70 \%$ de l'emploi salarié total. L'économie locale, vivrière et domestique, est basée sur une agriculture d'autosubsistance, complétée par de fortes importations. En dépit de la présence d'un aéroport international à Wallis, le tourisme demeure très limité.

Au contraire, le tourisme constitue un revenu important de la Polynésie française, avec la perliculture, la pêche ou la culture de la vanille. Mais l'économie polynésienne demeure fragile - le taux de chômage a doublé entre 2007 et 2012 et continue de reposer sur les dotations de l'État (Poirine, 2011), tandis que l'Institut de la statistique de la Polynésie française (ISPF) mesurait un taux de pauvreté en conditions de vie atteignant $28 \%$ en 2009 (17\% de taux de pauvreté en 2008 en Nouvelle-Calédonie) $)^{4}$, en relevant une répartition très inégale des revenus. Sur un territoire de 121 îles volcaniques ou coralliennes (dont 76 habitées) représentant une surface maritime de 2,5 millions de $\mathrm{km}^{2}$ pour $3814 \mathrm{~km}^{2}$ de terres émergées sur cinq archipels (Société, Tuamotu, Gambier, Australes, Marquises), la Polynésie française est dotée d'un atout océanique indéniable dans cette partie stratégique du monde. Sa population s'élevait à 270500 habitants en 2014, soit légèrement davantage que la Nouvelle-Calédonie, dont la densité de population est environ cinq fois inférieure. Ces brefs éléments généraux sur ces trois territoires permettent de situer leurs spécificités, qui n’excluent pas des échanges entre les territoires français de la région océanienne. Il existe par ailleurs des manifestations différentes du religieux dans les trois sociétés respectives, qui présentent de profondes particularités, avec des degrés variés de coutume, de tradition et de sacré qui donnent des accents singuliers au christianisme légué par la colonisation (Baubérot et Regnault, 2008).

En effet, les îles de Polynésie française, NouvelleCalédonie et Wallis-et-Futuna développent entre elles des relations déjà anciennes, ainsi qu'avec leurs voisins respectifs à l'échelle océanienne. Cet environnement régional s'avère disparate en fonction des territoires et des contextes propres à chacun de ces territoires : la Nouvelle-Calédonie, située à proximité de la puissance australienne, fait tampon avec «l'Océanie proche » et l'espace mélanésien, alors que la Polynésie française, très étendue, ferme la région océanienne à l'est, ses archipels s'étirant autour de " l'Océanie lointaine " dans la sphère d'influence polynésienne.

Certes, les problématiques varient selon les perspectives d'intégration régionale de ces territoires. Par leur proximité plus directe qu'avec Tahiti, la Nouvelle-Calédonie et Wallis-et-Futuna ont une relation d'échanges commerciaux et migratoires plus importante. Wallis-et-Futuna est située entre les deux autres territoires, à 2800 $\mathrm{km}$ de la Polynésie française et à $2000 \mathrm{~km}$ de la Nouvelle-Calédonie. Mais le "Caillou " constitue un pôle d'attractivité pour les Wallisiens, en raison de la différence d'échelle territoriale et économique entre les deux entités. La présence d'une importante diaspora wallisienne et futunienne en Nouvelle-Calédonie s'accompagne

4. Ces taux constituent des indicateurs aux méthodes différentes, qui possèdent toutefois de nombreux biais, dont l'estimation chiffrée de conditions de vie qui ne rendent pas vraiment compte d'un mode vie rural et d'une économie traditionnelle toujours présents, en particulier en dehors des zones urbaines de Papeete et de Nouméa, ainsi qu’à Walliset-Futuna. 
d'une communauté tahitienne et polynésienne qui a migré en fonction de critères socio-économiques. Le «boom du nickel» de 1969-1970 entraîna la migration de 15700 Européens, 3500 Tahitiens et 2900 Wallisiens et Futuniens, soit 24200 migrants entre 1969 et 1973. En comparaison, l'installation du Centre d'expérimentations du Pacifique en Polynésie française au début des années 1960 avait déjà connu une migration de 12200 personnes nées hors du territoire entre 1962 et 1971 . Si elle a contribué à la transformation de la société et de l'économie polynésiennes, la migration a été deux fois inférieure à celle se déroulant en Nouvelle-Calédonie sur une courte période. Les migrations européenne et polynésienne ont pu être perçues par les Mélanésiens comme une nouvelle colonisation (Rallu et Baudchon, 2003), accélérant la cristallisation de la revendication politique mélanésienne (Leblic, 1993, 2003 ; Soriano, 2014). L'émergence de mouvements autonomistes et/ ou indépendantistes en Nouvelle-Calédonie et en Polynésie française a développé un tissu de relations entre les acteurs de ces mouvements notamment engagés dans la lutte pour l'indépendance de leur territoire. Le processus s'est avéré chaotique en Nouvelle-Calédonie à la suite des " événements" (1984-1988). Des convergences politiques et militantes se sont nouées également au sujet connexe des protestations contre les expérimentations nucléaires conduites par la France à Moruroa et Fangataufa (Tuamotu, Polynésie française), qui focalisaient l'attention de la région à cette époque (Mrgudovic, 2008 ; Mohamed-Gaillard, 2010). Lopposition à la présence et aux essais atomiques français dans les années 19870 et 1980 a de la sorte renforcé le processus d'intégration régionale.

Les échanges intra-territoires français $\mathrm{du} \mathrm{Pa}$ cifique montrent-ils le développement d'une politique régionale cohérente de la France pour ces trois territoires? Il semble davantage que les convergences émergentes sur les plans politique et institutionnel s'inscrivent, d'une part, en tant que collectivité d'outre-mer (Сом) de la République française, mais aussi par l'association avec l'Union européenne en tant que Pays et Territoire d'outre-mer (ртом), qui favorise les pratiques d'intégration régionale en matière économique, scientifique ${ }^{5}$ et culturelle. Les Ртом (les territoires français et Pitcairn dépendant du Royaume-Uni) représentent ainsi une "présence européenne " dans la région Pacifique, selon les termes de l'ue dans le document présentant en 2006 la stratégie de renforcement du partenariat entre l'Ue et les États ACP - Afrique, Caraïbes, Pacifique - (Choi, 2010). L'intégration régionale ne s'apparente donc pas à un "serpent de mer" ou à une marotte institutionnelle, mais s'inscrit dans une politique française et européenne de consolidation de leur présence stratégique, bien que les limites de ces considérations géopolitiques soient diplomatiquement connues et évaluées depuis longtemps (Regnault, 2013: 235-244).

Un autre aspect relatif à l'intégration régionale concerne directement l'intégration territoriale au sein de chacun des territoires, dont les découpages administratifs sont distincts. La régionalisation dans les territoires concerne essentiellement la Nouvelle-Calédonie (Leblic, 1993 ; 2004). Cette option institutionnelle a été proposée sous des formes et des finalités différentes par le gouvernement central pour résoudre la question calédonienne, dans l'optique de diviser la revendication nationaliste kanak à la suite de la crise et de violences politiques entre les militants indépendantistes kanak et les loyalistes. Elle s'est avérée inopérante entre 1985 et 1988. Mais ce mouvement a été suivi par la provincialisation, issue des accords de Matignon en 1988 (Leblic, 1993 ; Faberon, 2003). Des représentants des trois provinces (Nord, Sud et îles Loyauté) siègent au Congrès de NouvelleCalédonie, où ils élisent un gouvernement. Pour autant, le découpage aux formes fédérales des provinces de Nouvelle-Calédonie (Faberon, 2008) ou l'organisation en circonscriptions administratives divisées en districts subdivisés en village dirigés par des chefs à Wallis-et-Futuna (Soulé, 2003 ; Guiot et Leblic (éds), JSO 122-123, 2006 ; Argounès et al., 2011 : 46) se différencient de la force centralisatrice de Papeete et de Tahiti en Polynésie française (Merceron et Morschel, 2013) pour y déceler des convergences en termes de régionalisation interne ${ }^{6}$. En outre, la Polynésie française, par son étendue maritime, représente presque à elle seule un espace régional, avec 121 îles étirées sur une surface comparable à celle de l'Europe occidentale. Ces territoires à la géographie, à l'histoire et aux institutions différentes possèdent tout de même quelques éléments propices au développement de leurs relations.

Des échanges culturels mais aussi institutionnels, ministériels et économiques se nouent entre la Nouvelle-Calédonie et la Polynésie française. Au niveau des relations économiques entre les territoires, quelques facteurs de convergence apparaissent au regard de missions ministérielles entre Tahiti et la Nouvelle-Calédonie. Ils concernent la gestion d'un tourisme durable

5. Voir, par exemple, le projet d'intégration régionale des pays et territoires d'outre-mer européens autour de la gestion intégrée des zones côtières (INTEGRE), dans le cadre du $\mathrm{X}^{\mathrm{e}}$ FED régional PTOM Pacifique de l'Union européenne et, dans ce dossier, l'article de Rémy-Louis Budoc (pp. 21-38), qui offre un aperçu complet de ces différents programmes.

6. En dépit de quelques tentatives, dont celle du secrétaire d'État aux ром-том Georges Lemoine (1983-1986), qui envisageait de "régionaliser la Polynésie française " (in Regnault, 2003 : 114). 


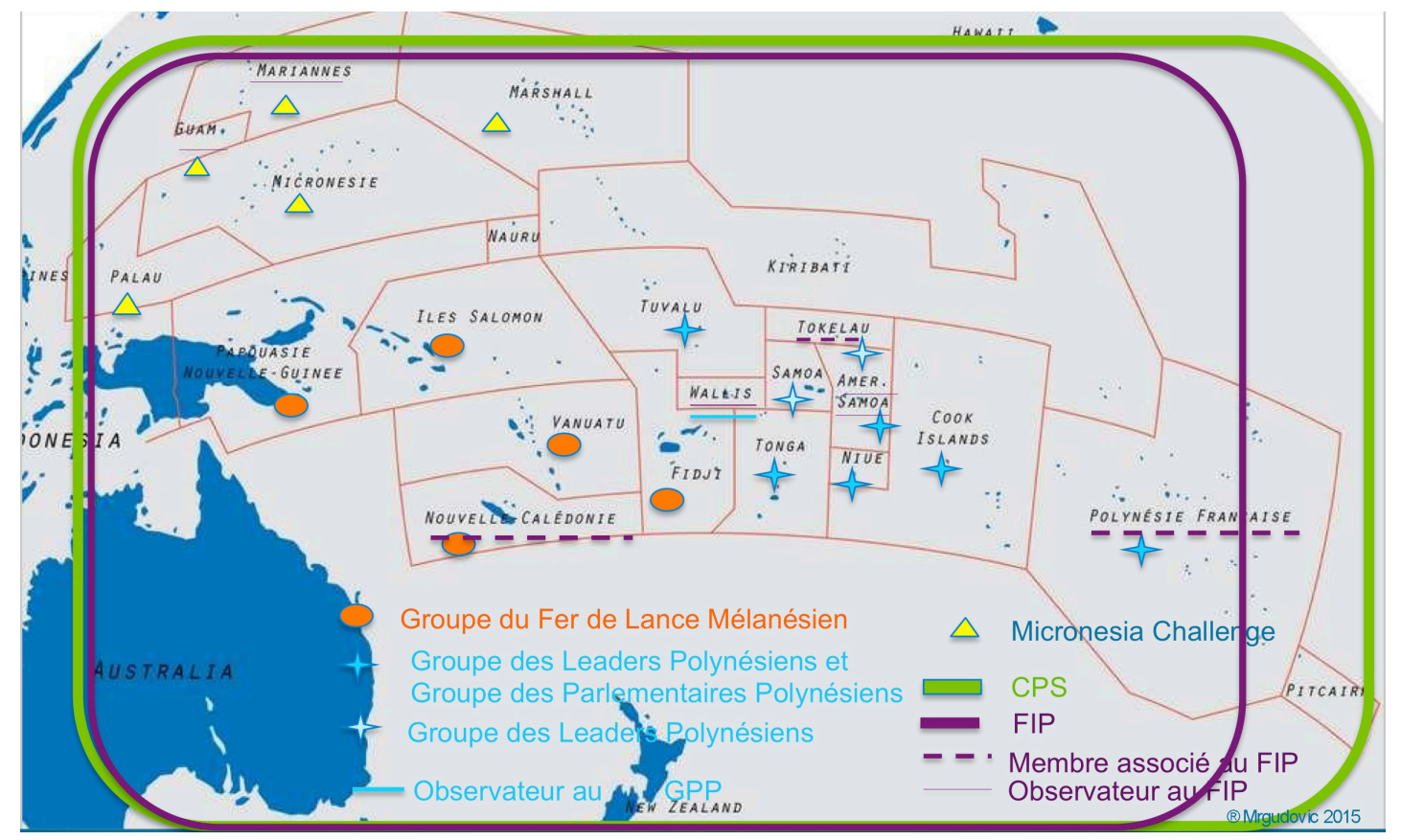

CARTE 2. - Intégration régionale en Océanie (@ http://www.defense.gouv.fr/var/dicod/storage/images/basede-medias/images/marine/photos-des-breves/carte-zee/2067692-1-fre-FR/carte-zee.jpg pour le fond de carte \& Nathalie Mrgudovic)

(La Dépêche de Tahiti, 24 février 2015), ou la mise en place de relations entre les Chambres de commerce et d'industrie et les organisations patronales et syndicales sur les questions du lobbying en faveur de la défiscalisation ( $"$ Les trois collectivités françaises du Pacifique s'unissent ", La Dépêche de Tahiti, 30 octobre 2014). Ce dernier aspect représente un sujet de convergence entre les autorités économiques des collectivités d'outre-mer dans leur ensemble ${ }^{7}$. Elles appellent à la prise en compte du potentiel de la ZEE (David, 2008) par les parlementaires français, notamment lors de la conférence sur les collectivités d'outre-mer du Pacifique et les enjeux stratégiques et économiques de la région qui s'est tenue à l'Assemblée nationale en novembre 2014. Sous un angle économique, le processus d'intégration régionale révèle les impacts positifs des accords commerciaux intra-régionaux sur les flux d'échanges entre les pays membres. Ce type d'étude met aussi en relief le legs historique de la colonisation dans la structuration des échanges (Ro’i, 2013). Avant le développement récent des échanges commerciaux, économiques et technocratiques entre le Fenua (" pays ») et le Caillou, il a longtemps été considéré que la distance (4 700 $\mathrm{km}$ ) entre les deux territoires, des histoires et des sociétés différentes ne favorisaient pas une intégration économique mutuellement intéressante. A l'exception de quelques échanges patronaux, il y a peu d'échanges commerciaux, car les " clans familiaux" verrouillent le business à Nouméa. La production serait trop faible en Polynésie française, selon l'avis de l'ancien président du Rassemblement pour la Calédonie dans la République (RPCR), Jacques Lafleur, dont l'entreprise politique concurrente au sein des relais chiraquiens au RPR avec le leader polynésien Gaston Flosse mettait en scène la rivalité entre les deux territoires dans les années 1980 et 1990, chacun briguant par exemple le poste de Secrétaire d'État au Pacifique Sud dans le gouvernement Chirac en 1986 :

«On est totalement différents. On peut avoir des échanges politiques. S'agissant des échanges économiques, ça se fera à sens unique parce que la Polynésie ne produit pas grand-chose." (Lafleur, in Kotra, 2009: 100)

Certes, la complémentarité économique entre la Polynésie française et la Nouvelle-Calédonie est loin d'être flagrante a priori, mais les échanges entre les deux territoires n'ont pas exclusivement vocation à être seulement de nature politique ou économique. En effet, l'échange de connaissances et de savoirs, par exemple lors du Festival international du film océanien (FIFO), démontre ainsi la richesse des échanges culturels qui naissent des créations artistiques par les acteurs des différents territoires océaniens. 


\section{Degrés et natures variés des échelles de l'intégration régionale}

Les différents niveaux de l'intégration régionale autour des entités françaises sont articulés autour de trois dimensions : l'intégration entre les territoires français, l'intégration avec les autres territoires et l'intégration dans des groupes régionaux, encouragée par les acteurs extérieurs en fonction de l'architecture institutionnelle de la région océanienne.

Les phénomènes d'intégration régionale se présentent principalement sous la forme de coopérations aux formes diverses. Celles des entités françaises avec leurs territoires voisins et/ou les organisations régionales concernent aussi bien les coopérations militaires (formation, surveillance des ZEE, interventions humanitaires), scientifiques (environnement [Trichet et Leblic éds, 2008]), changements climatiques ; échange de savoirs/technologies aquacoles, agricoles ou minières ; structuration du réseau de chercheurs e-toile Pacifique, etc.), ou les champs économiques (import/export; aquaculture; économie numérique) et culturels (manifestations sportives, médiatiques, artistiques).

Après 1945, les premières formes de coopération entre territoires de la région ont amorcé des processus d'intégration régionale, qui se sont traduits sur les plans institutionnel et politique par la création d'organisations régionales et subrégionales. Dès 1947, la commission du Pacifique Sud - devenue Communauté du Pacifique en 1997 - est instaurée. Tandis que le Forum du Pacifique - créé en 1971 à Wellington (Nouvelle-Zélande), devenu le Forum des îles du Pacifique en 2000 -, ou le groupe du Fer de Lance mélanésien (GFLM), établi en 1988 à Port-Vila (Vanuatu), sont mis en ouvre ultérieurement dans des contextes différents: lors de la phase de décolonisation des territoires du Commonwealth marquée par la contestation des essais nucléaires français dans les années 1970 pour le Forum ; et, lors de la crise calédonienne des années 1980 pour le GFLM, même si cette organisation politique entre États mélanésiens a depuis mis l'accent sur l'aspect économique de leur coopération.

Les rapports avec le Front de libération nationale kanak socialiste (FLNKS), qui a été intégré dans l'organisation mélanésienne depuis mars 1990, montre toutefois le caractère politique premier d'un groupe, qui a œuvré au développement d'un "sentiment régional " mélanésien. La recherche d'une intégration économique a ensuite été illustrée par l'intégration de la Nouvelle-Calédonie au marché de libre-échange mélanésien le 3 juillet 1999, lors du XIII ${ }^{\mathrm{e}}$ sommet du GFLM à Port-Vila. Cet accord a été rendu possible par la signature de l'accord de Nouméa le 5 mai 1998 entre le FLNKs, le RPCR et le gouvernement français. En outre, le 15 août 2000, la Nouvelle-Calédonie est admise pour la première fois à participer aux travaux post-forum du Xxxir ${ }^{\mathrm{e}}$ Forum du Pacifique à Nauru. Ainsi, les 15 août 2002 et 16 août 2003 , le président du gouvernement de Nouvelle-Calédonie, puis une délégation FLNKS-RPCR, participent directement au Forum du Pacifique à Suva (Fidji) puis à Auckland (voir notamment Mrgudovic, 2003 ; Mohamed-Gaillard, 2011 ; Tabani, 2011 ; Graff, 2012). Le Forum, dont les membres sont signataires des accords ACP/UE, représente de plus un appui pour la politique régionale de l'UE, qui est intéressante pour les signataires par son flux d'aides techniques et financières aux îles du Pacifique (Fabry, 2005).

Le régionalisme mélanésien a également des relations polynésiennes, en particulier avec le parti indépendantiste tahitien, le Tavini Huiraatira d'Oscar Temaru. Lors du $\mathrm{Xv}^{\mathrm{e}}$ sommet du GFLM aux Salomon, le 6 août 2003, Temaru était l'invité spécial pour la réinscription de la Polynésie française sur la liste onusienne des pays à décoloniser. Le GFLM a continué de soutenir sa démarche, lorsqu'en mars 2011, le président du groupe du Fer de Lance écrivit au président du forum pour affirmer son soutien aux indépendantistes tahitiens (Regnault, 2013: 199). De son côté, le Forum rappelle sa décision de 2004 qui reconnait le principe d'auto-détermination, mais encourage la Polynésie française et la France à résoudre cette question par une approche interne et consensuelle (Regnault, 2013:201).

Si la Communauté du Pacifique représente une entité davantage technique, la raison de la création du forum des îles du Pacifique était essentiellement politique. D’ailleurs, le régionalisme océanien s'est développé en rapport avec le mouvement de décolonisation. La posture régionale a été ainsi structurée par le rejet de la politique de la France lors de la crise calédonienne et de la persistance des essais nucléaires (MohamedGaillard, 2012). Il en est de même du GFLM et, plus récemment, des deux groupes de dirigeants et de parlementaires polynésiens, analysés ici par Sémir Al Wardi (pp. 75-88).

Déjà, à la suite de la mobilisation du Fer de Lance au milieu des années 1980, le président du gouvernement de la Polynésie française, Gaston Flosse, avait émis l'idée en septembre 1985 lors d'une visite de Jacques Chirac à Tahiti, de lancer une "fédération des États polynésiens " pour répondre au mouvement mélanésien (Bessard, 2013 : 327). Ironie de l'histoire, en août 2013, redevenu président de la Polynésie française, Gaston Flosse est élu par ses pairs, en tant qu’aîné, comme président du Pacific Leaders Group, regroupant huit "pays» polynésiens. Cette initiative s'avère moins formelle que l'instauration d'une fédération, mais elle s'inscrit ce- 
pendant dans la recherche d'un groupe régional polynésien visant à contrebalancer l'influence des organisations mélanésiennes. En 1992, lors du Iv Festival des Arts du Pacifique, le président du gouvernement de Polynésie française, Gaston Flosse, déclara, à l'occasion d'un rassemblement de grandes pirogues :

" Nous sommes un seul et même peuple. » (Bessard, $2013: 306)$

L'orientation de sa diplomatie privilégie surtout le versant polynésien des relations océaniennes, en mettant en avant la dimension familiale rapprochant les « frères et sœurs du Pacifique ». Lors du sommet France-Océanie à Tahiti en août 2003, le vice-président de la Polynésie française et ancien gendre de Gaston Flosse, Édouard Fritch, mandaté pour transmettre l'invitation de la France aux dirigeants océaniens, relevait que ces derniers avaient salué cette initiative :

"Certains ont même parlé d'événement historique, qu'un membre de la famille ait ce souci de rassembler les frères et les sœurs du Pacifique [...]. » (Bessard, $2013: 306$ )

Par ailleurs, les territoires français du Pacifique sont inscrits dans la politique de la France dans le Pacifique, dont la consistance fluctue... Mais ils relèvent aussi de la politique de l'Union européenne, en tant que pays et territoire d'outremer (PTOM) associés à l'UE, leur permettant ainsi de postuler à l'attribution des fonds européens de développement (FED). Le principal bailleur de fonds de ces territoires demeure cependant l'État français. Néanmoins, le rôle des acteurs " extérieurs ", l'Union européenne en particulier, participe au développement d'un processus d'intégration régionale qui fait partie des référentiels des politiques publiques européennes d'aide au développement. Au même titre que la "bonne gouvernance " ou le "développement durable ", le renforcement des capacités en matière $d$ ' " intégration régionale " est mis en avant dans les programmes de coopération internationale de l'Union européenne. Toutefois, elles ne figurent pas au rang des conditionnalités politiques qui accompagnent parfois l'aide financière et technique apportée par l'UE, même si celle-ci cherche à remédier à la faible intégration régionale des outre-mers français dans leur zone (Fabry, 2005). Larticle de Rémy-Louis Budoc (pp. 2138) éclaire particulièrement la dimension euro- péenne dans le processus d'intégration régionale autour des trois territoires français du Pacifique. Enfin, la création en 2002 de l'OCTA, association des PTOM de l'ue, vise à assurer la défense des intérêts des autorités insulaires auprès des instances européennes, bien que leur dispersion et leur hétérogénéité semblent les desservir face au bloc technocratique européen.

\section{Un concept d'intégration régionale vecteur d'ambiguïtés}

Le concept d'intégration régionale est conçu de façon souple, sans entrer dans les débats théoriques relatifs au néo-fonctionnalisme ${ }^{8}$ ou au néo-régionalisme sur la question de l'intégration régionale dans les théories des relations internationales. Ces débats s'avèrent occidentalo-centrés, tandis que les études prennent majoritairement appui sur les États (Dabène, 2009). Non seulement le cadre qui nous préoccupe comprend des territoires aux degrés de souveraineté et aux statuts multiples, mais le contexte d'étude souvent lié à la dynamique de la construction de l'Union européenne, qui préside au développement de ces théories, n’offre pas une applicabilité conceptuelle vraiment intéressante dès lors que l'on étudie le contexte océanien. Confronté aux phénomènes d'intégration régionale en Amérique latine, le politiste Olivier Dabène propose une autre définition de l'intégration régionale,

\begin{abstract}
«[... En termes de processus historique de hausse du niveau d'interaction entre unités politiques (infranationales, nationales ou supranationales), provoquée par des acteurs partageant des idées, arrêtant des objectifs et trouvant des instruments pour les atteindre, et ce faisant contribuant de façon volontaire ou involontaire à construire une région. » (Dabène, 2009 : http://www.sciencespo.fr/opalc/content/integrationet-democratie)
\end{abstract}

Cette approche englobe la diversité des acteurs (publics et privés), des registres (formels et informels) et des niveaux d'interaction du processus, qui résulte soit d'une stratégie délibérée soit d'un effet émergent, pouvant entraîner la création d'institutions. Par exemple, l'idée d'une Pacific Way (Mara, 1997) faisait sens pour ses protagonistes, à partir des idées de recherche du consensus et de solidarité entre les peuples du Pacifique. Elle avait notamment pour objectif la formation

8. Les théories fonctionnalistes ou néo-fonctionnalistes ont été élaborées au même moment que la construction européenne s'est développée. L'attention portée sur ce processus de régionalisation a conduit à occulter d'autres cas de régionalisation dans d'autres parties du monde.

9. Les tenants du néo-régionalisme critiquent la dimension unidimensionnelle et restreinte du régionalisme analysé par les fonctionnalistes, c'est-à-dire une démarche consistant à décrire le processus d'intégration européenne, puis à généraliser ses spécificités comme un modèle standard servant à évaluer les autres processus de régionalisation à l'œuvre ailleurs (Breslin et al., 2002). 
d'un régionalisme, dont l'un des instruments fut le Forum du Pacifique Sud, qui voulait trancher avec le rôle plus feutré et limité en matière politique de la Commission du Pacifique Sud.

La région peut être comprise comme un mode de régulation et d'organisation des rapports entre les différents acteurs afin de les consolider et de les faciliter. Il ne s'agit pas d'un simple cadre d'échanges entre acteurs, mais plutôt d'un construit (Van Langenhove, 2003). Le renouveau de l'analyse du concept de région et de régionalisme met en évidence la région comme une nouvelle forme d'entité politique, à la fois multiculturelle, multidimensionnelle et dynamique, car elle se trouve inscrite dans un processus complexe de construction résultant de la volonté des acteurs. Le régionalisme n'a donc pas seulement pour vocation d'intensifier les relations économiques mais de donner "un sens » régional aux différentes entreprises politiques, sociales et culturelles (Hau'Ofa, 2015). Un tel régionalisme est traversé par des flux concernant des acteurs aux multiples profils sociologiques, qui ne sont pas uniquement les " acteurs d'une intégration par le haut ", celle des dirigeants politiques, mais concernent aussi par exemple les acteurs culturels et économiques. Ainsi, l'" intégration par le bas " (Bach, 1998) met en exergue des mouvements de coopération, de transaction et d'interaction transfrontalières lancés par et impliquant les acteurs non-étatiques, par exemple les associations de femmes, souvent à l'origine d'initiatives culturelles dans la région (Cerf, 2007). Malgré tout, les rapports de force économiques penchent en faveur des territoires français en comparaison de leurs voisins océaniens, à l'exception des puissances régionales australasiennes, dans la mesure où la Nouvelle-Calédonie, et à un degré moindre, la Polynésie française, ont les PIB par habitant parmi les plus élevés de la région, presque au niveau de la Nouvelle-Zélande. Or, développer des relations commerciales avec de petits marchés, peu solvables et produisant les mêmes choses à moindre coût, est d'un intérêt économique relatif (Gay, 2008 : 106).

\section{L'intégration régionale, des legs coloniaux aux flux mondialisés}

Le développement des échanges et l'identification régionale à un ensemble de traits culturels qui font sens dans le Pacifique Sud comportent en effet une dynamique de "retrouvailles familiales postcoloniales » en quelque sorte, qui est progressivement alimentée par des échanges de nature variée. La diversité géographique, culturelle, politique et économique sépare en apparence les territoires et entités du Pacifique Sud. Or, un mouvement contemporain de "renaissance " de l'intégration régionale, en écho à l'histoire préco- loniale de la région "océanienne " marquée par de nombreux échanges inter-insulaires, amène à réinterroger les relations intra- et extra-territoriales des entités françaises du Pacifique Sud.

Malgré tout, le processus de régionalisation s'inscrit dans le temps long des relations au sein du «continent d'îles ». D'ailleurs, les flux de l'époque précoloniale ont-ils des correspondances à l'origine de certains développements relationnels entre territoires, par exemple entre les "pays polynésiens " ? Si le Pacifique d'avant le contact avec les Européens n'était pas véritablement un espace de culture globale, en dépit du "complexe culturel lapita " (Sand, 2010; Conte, 2013 : 28) formant un substrat culturel commun à ces peuples, les populations de l'Océanie lointaine, principalement celles du triangle polynésien (Hawaï-île de Pâques-Nouvelle-Zélande), ont conservé entre elles des relations durant des siècles. Elles présentaient une "grande proximité culturelle qui autorise à les inclure dans un espace culturel global " (Conte, 2013 : 29). Néanmoins, selon l'archéologue Éric Conte, il semblerait aventureux de considérer la formation récente de groupes de représentants politiques du monde polynésien comme une réminiscence contemporaine de relations anciennes - mythe d'un âge d'or des relations précoloniales -, qui auraient été éclipsées par la période coloniale.

Marquées par le legs des logiques coloniales, les fragmentations régionales océaniennes sont repérables dans les divisions de l'Océanie conventionnellement acceptées (Tcherkézoff, 2009), dans le découpage territorial et le mode d'administration, qui a fait émerger des systèmes juridiques et administratifs différents en fonction des puissances coloniales. Les territoires français sont restés relativement isolés de leurs voisins, en particulier en raison des rivalités coloniales et religieuses. Les enjeux stratégiques et économiques dans la deuxième moitié du $\mathrm{xx}^{\mathrm{e}}$ siècle, dans le cadre d'une politique archipélagique de puissance (Mohamed-Gaillard, 2010), n’ont guère favorisé une ouverture régionale de ces territoires régis en vertu des "enjeux de puissance" (Mrgudovic, 2008). En Afrique, les États européens avaient cherché à décourager l'entrée et la sortie de marchandises durant la période coloniale, afin de contrôler voire limiter les contacts entre acteurs économiques faisant partie d'empires coloniaux différents (Grant et Söderbaum, 2003 ; Meyer, 2006 : 337). Le processus d'intégration régionale est de fait influencé par les types de relations et d'échanges qui se sont développés depuis lors. On note l'importance des facteurs exogènes dans un processus régional qui n'a rien de téléologique, en cela qu'il marquerait un stade d'avancée vers la libération graduelle de l'économie mondiale - le régionalisme comme "étape vers la libéralisation internationale " (Meyer, $2006: 307$ ) - en vertu de 
la logique libérale qui sied à la plupart des théories relatives au régionalisme. Or, un changement de considération politique de la France envers ses territoires tend aujourd'hui à favoriser l'intégration régionale. Auparavant, elle veillait à tenir ses COM à l'écart des influences régionales, même de manière rigide au sein des institutions régionales dont la Commission du Pacifique Sud. Pourtant, cela n'a pas empêché des rapprochements entre mouvements autonomistes et indépendantistes des territoires français du Pacifique, entre eux mais aussi avec des leaders et des mouvements politiques régionaux qui les soutiennent. Dès lors, les autorités françaises encouragent désormais le processus d'intégration régionale et la participation de ses territoires aux instances régionales. Cela lui permet non seulement d'améliorer son image régionale, mais aussi de gêner l'extériorisation des groupes politiques en faveur de la décolonisation onusienne (Mohamed-Gaillard, 2010).

Une autre approche consiste à entrevoir l'intégration régionale comme un facteur de réduction de la dépendance économique productrice d'inégalité, ou extraversion (Bayart, 1999). Une trop forte ouverture au marché international (Hugon, 2003) sous l'influence de l'organisation mondiale du Commerce (OMC), a peu d'intérêt économique pour les territoires océaniens. Mais une vision moins centrée sur l'économie laisserait aussi entrevoir la volonté des acteurs océaniens de se retrouver " entre soi ", de renouer et de développer des relations aux accents régionaux, qui peuvent faire davantage sens, en comparaison de l'imposition de relations lointaines et unilatérales qui prévalaient entre de nombreux territoires océaniens et les puissances coloniales. Quant aux États insulaires indépendants, l'intérêt de la régionalisation pour des États susceptibles d'être marginalisés économiquement au niveau mondial (Giesen, 2011) possède une dimension commerciale limitée mais n'est pas exempt d'avantages politique. Les théories institutionnelles du régionalisme montrent que l'institutionnalisation et l'harmonisation des politiques s'avèrent des facteurs de réduction des incertitudes et de stabilité des interactions entre les acteurs. Il s'agit moins de simplement encourager les liens commerciaux, mais plutôt de développer des politiques sectorielles (environnement, culture, santé). Ce mouvement d'autoprotection serait un contre-mouvement moderne vis-à-vis de la mondialisation selon Björn Hettne (2000). Celui-ci reprend l'analyse de Karl Polanyi relative à la libéralisation de l'économie; ce "premier mouvement " entraîne un contre-mouvement social dans un second temps. Toutefois, peut-on aller aussi loin dans la conceptualisation de l'intégration régionale autour des entités françaises du Pacifique?

Le voisinage des entités françaises du Pacifique Sud, d'un point de vue maritime, n'offre pas de perspectives d'intégration régionale très poussées au sens occidentalo-centré d'un processus essentiellement politique relatif à un transfert de souveraineté - souvent fraîchement acquise pour maints États de la région -, à l'instauration d'un marché commun et d'une union douanière. La Nouvelle-Calédonie se situe entre l'Australie et Fidji, tout en étant proche du Vanuatu et des îles Salomon. Wallis-et-Futuna est dans une position océanienne relativement centrale, mais sa taille et son défaut d'infrastructures ne facilitent guère des échanges plus réguliers avec ses voisins (Tuvalu, Tokelau, Samoa, Tonga ou Fidji, voisin le plus proche à $280 \mathrm{~km}$ au sud-ouest de Futuna). Alors que Wallis se rattachait autrefois au modèle tongien, Futuna s'inspirait davantage du cas samoan. La colonisation a modifié les dynamiques régionales de ces territoires pour ne faire de ces trois royaumes qu'une seule entité politique (Leblic et Guiot, 2004 ; Angleviel, 2006). La Polynésie française, en périphérie orientale de l'Océanie, est voisine "directe » de territoires aux statuts différents : Pitcairn (Royaume-Uni), Kiribati (État indépendant) et les îles Cook (pays associé à la Nouvelle-Zélande). Elle se situe surtout au centre du triangle polynésien NouvelleZélande-Hawaï-île de Pâques, et presque au milieu d'un axe imaginaire entre l'Asie et l'Amérique du Sud. Pourtant, le potentiel d'échanges entre les territoires et leur périphérie, qui se traduit par exemple par la participation croissante de ces entités aux institutions régionales, appartient à une dynamique d'intégration régionale originale, autour d'une architecture transnationale de coopération. Il en résulte un régio-multilatéralisme basé sur un équilibre des institutions régionales océaniennes, qui se trouve dans une phase de redéfinition, posant la question de la verticalisation des organisations régionales et de leur démocratisation (Lechervy, 2015).

À l'évidence, cette intégration ne correspond pas au schéma ou au degré de celle qui préside à la formation de l'Union européenne. Le modèle de l'intégration régionale tend à être enrichi par une vision océanienne qui ne place pas nécessairement l'économie et la concurrence non faussée comme socles d'une identité régionale en formation et fondée sur un rapport au temps et à l'océan qui réinvente une forme d'intégration régionale au sein d'un espace océanique (Hau'Ofa, 2015). Par exemple, l'idée d'une "Union Pacifique " ou d'une "Union océanienne", telle qu'elle est formulée par le sénateur souverainiste polynésien Richard Tuheiava, représente selon

10. Thème d'un programme de recherche du laboratoire Gouvernance et développement insulaire (GDI), Université de Polynésie française (2015-2017), dont les auteurs font partie. 
lui un "projet fédérateur ", qui pourrait prendre appui sur la francophonie ainsi que sur une vision commune océanienne, offrant à la Polynésie française une place centrale dans un processus communautaire régional en gestation (Massau, 2011 : 152-160). Enfin, l'intégration régionale pose la question de la sécurité militaire dans une Océanie de plus en plus convoitée ${ }^{10}$; mais ce problème concerne moins directement ce dossier relatif aux territoires français du Pacifique, étant donné la présence de l'armée française et la configuration institutionnelle qui prévaut aujourd'hui. Cependant, le déplacement des enjeux dans la zone apporte son lot de nouvelles problématiques, par exemple celle de la montée en puissance des pays asiatiques dans la région - parfois relayée par les diasporas asiatiques présentes dans les territoires français (Fer, 2014) -, et en corollaire, la surveillance des pratiques de pêches commerciales (Chine, Taiwan, Japon, Corée du Sud ; Crocombe, 2007 ; David, 2008, 2011). Les navires jouent de l'immensité océanique pour échapper aux réglementations, prolongeant différentes formes d'écocide, dont les requins sont notamment les témoins malencontreux et des victimes rémunératrices.

L'intégration régionale autour des territoires français se situe donc au carrefour d'enjeux internationaux multiples, dont de nombreuses facettes présentent des mutations significatives : l'enjeu de l'ouverture économique d'un espace marginal dans la zone Asie-Pacifique, la révolution technologique et les problématiques du changement climatique. Par exemple, en 2010, la pose du câble sous-marin en fibre optique $\mathrm{Ho}$ notua ("lien du large" ou " colonne dorsale») entre Tahiti et Hawaï, en reliant la Polynésie française au reste du réseau mondial de télécommunications, a illustré l'insertion de ce territoire dans la mondialisation et a ouvert le champ des potentialités de l'économie numérique (Laborie, 2013). L'essor d'internet et des technologies numériques transcende l'éparpillement et l'isolement géographiques des îles océaniennes. Il favorise les échanges régionaux en les facilitant. L'extension du câble reste cependant en suspens ("Le câble dans l'impasse ", La Dépêche de Tahiti, 17 janvier 2015) malgré de nombreux projets, dont celui d'un câble reliant la Polynésie française et la Nouvelle-Calédonie, elle-même connectée au réseau australien (Argounès et al., 2011). En outre, l'enjeu environnemental, fondamental en Océanie, nécessite des coopérations à l'échelle régionale afin de répondre à l'interdépendance des périls écologiques. Face à ces multiples transformations globales, l'horizon d'un océan de nouvelles opportunités et de nouvelles contraintes, qui se dessine progressivement pour ces territoires, montre que les marges de coopération, au sein de la francophonie du Pacifique et par-delà le francocentrisme, demeurent très importantes en Océanie. Dans la « Françocéanité ", l'appropriation des nouvelles compétences internationales et régionales par les gouvernements calédonien et polynésien permet progressivement l'élaboration de stratégies territoriales en accord avec les autorités de la République (Lechervy, pp. 105-122).

Â partir des dynamiques contemporaines d'institutionnalisation des échanges régionaux entre les territoires océaniens, des relations naissent ou renaissent, tendent à prendre de nouvelles formes politiques, économiques, sociales ou culturelles. De fait, les formes du processus d'intégration régionale sont appréhendées au regard de plusieurs perspectives disciplinaires et thématiques.

\section{Un dossier aux études hétéroclites sur les dynamiques régionales}

L'analyse des relations entre l'Union européenne (UE) et les pays et territoires d'Outre-Mer (PTOM), sujet de l'article de Rémy-Louis Budoc (pp. 2138) offre une première perspective intéressante dans ce dossier pour pénétrer les arcanes institutionnelles de l'intégration régionale au prisme du rôle de l'ue, qui " a fait de la région Pacifique une nouvelle priorité de sa politique étrangère " (p. 23). L'auteur se réfère à la dernière décision d'association d'Outre-mer (DAO) adoptée le 23 novembre 2013, afin d'étudier les objectifs de modernisation des relations PTOM-UE, qui implique le développement des Ртом tout en différenciant cette nouvelle politique de celle "traditionnelle " de l'ue de coopération au développement. Ce " changement de cap dans les relations UE-PTOM » implique notamment un soutien à la politique d'intégration régionale des trois territoires français dans leur environnement.

L'intérêt de l'Union européenne dans la région a en particulier été développé par la France. En effet, la politique d'insertion menée par la France pour ses territoires du Pacifique Sud, mise en lumière dans l'article de Denise Fisher (pp. 3948), montre que la France est certainement parvenue, notamment grâce à l'arrêt des essais nucléaires en Polynésie française et à la mise en place des accords de Matignon-Oudinot puis de Nouméa en Nouvelle-Calédonie, à ce que ses territoires océaniens deviennent de plus en plus les acteurs de leur propre insertion régionale et participent aux activités des organisations régionales, ainsi quaux programmes de coopération militaire, d'échanges culturels ou encore sportifs. Pourtant, selon Denise Fisher, l'influence que la France exerce sur ses territoires est encore trop grande pour leur permettre de véritablement s'intégrer à la structure régionale.

Ainsi, dans son évaluation de l'état de l'architecture régionale, l'article de Tess Newton Cain 
(pp. 49-58) met en parallèle l'évolution des organisations régionales, qui semblent faillir à réformer une architecture régionale, et l'émergence d'un sous-régionalisme plus directement focalisé sur les questions de politique économique. L'auteur considère principalement les organisations régionales composant le Conseil régional des organisations du Pacifique (CROP), la Communauté du Pacifique et le Forum des îles du Pacifique. Le Pacific Plan, initié en 2005 pour renforcer cette architecture régionale, a déjà fait l'objet d'un premier bilan qui confirme que la région doit conserver sa structure essentiellement bicéphale et s'organiser autour de deux piliers principaux, l'un politique et l'autre technique. Face à ce mouvement de reconstruction régionale, le sous-régionalisme semble être accompagné par la défense d'intérêts insulaires communs. Ainsi, l'accord de Nauru conclu en 2010 sur la gestion de l'activité halieutique qui stipule qu'à

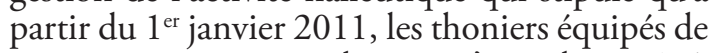
senne tournante et coulissante n'ont plus accès à l'une des zones de pêche les plus riches en thon tropical dans le monde, apparait plus attractif que l'organisation régionale (Agence du Forum des îles du Pacifique sur la Pêche). Les huit pays insulaires du Pacifique signataires de l'accord de Nauru (Papouasie Nouvelle-Guinée, Palau, îles Salomon, Etats fédérés de Micronésie, Kiribati, Nauru, Tuvalu et îles Marshall) forment dorénavant un véritable cartel du thon. Toutefois, Tess Newton Cain souligne les tensions qui peuvent exister entre intérêts nationaux et régionaux, par exemple au sujet de l'Université du Pacifique. Enfin, la place à accorder aux territoires français et américains au sein de l'architecture régionale n'est toujours pas vraiment réglée, ce qui contribue à cette perpétuelle mouvance du régionalisme dans le Pacifique.

Explorant le sub-régionalisme dans le Pacifique Sud, Nathalie Mrgudovic (pp. 59-74) analyse le bilan que le Groupe du Fer de Lance mélanésien (GFLM) peut tirer, vingt-cinq ans après sa création en mars 1988 à Port-Vila (Vanuatu). Initialement conçu pour porter le projet de construction nationale des nouveaux États mélanésiens (PNG, Salomon, Vanuatu) et peser davantage dans la balance régionale, le groupe entendait s'appuyer sur des valeurs culturelles communes fondées sur la Kastom, même si les aspirations des leaders mélanésiens ont parfois divergé. La volonté de promouvoir l'émancipation politique des peuples mélanésiens s'est traduit par le soutien du GFLM au mouvement indépendantiste kanak - le FLNKS - qui a par la suite été invité à devenir membre du groupe. L'objectif économique, rappelant vaguement le concept de "socialisme mélanésien ", entendait mettre en place un accord de libreéchange mélanésien. Mais la limite des produits à échanger, ainsi que le refus, jusqu'en 1998, de Fidji de rejoindre le groupe, a mis à mal la réa- lisation de ce projet, de même que le refus du Fer de Lance de soutenir le mouvement indépendantiste en Papouasie occidentale. Nathalie Mrgudovic souligne ensuite le rôle ambigu de Fidji auprès du groupe, car s'il a pu fragiliser le groupe par son absence, il l'a aussi, tout à la fois, renforcé et menacé par sa présence. Dès lors, l'auteur se demande d'une part, si le GFLM est devenu un rival ou au contraire un complément des organisations régionales, et tout particulièrement du Forum des Iles du Pacifique, et d'autre part, dans quelle direction le groupe saura poursuivre son évolution après la présidence tournante de Frank Bainimarama, Premier ministre fidjien.

Une autre manifestation du sub-régionalisme océanien, étudiée par Sémir Al Wardi (pp. 7588), concerne la formation du groupe des parlementaires polynésiens (GPP), créé en mars 2013. Après plusieurs tentatives, les États et territoires polynésiens sont parvenus à un rapprochement tout en se distinguant d'un autre groupe : le groupe des leaders polynésiens (GLP). Celui-ci, apparu deux ans plus tôt à l'initiative de Gaston Flosse, rassemble les leaders polynésiens. En procédant à une comparaison de ces deux groupes, l'auteur souligne l'absence de deux «États figurant dans le triangle polynésien à savoir les plus puissants : les États-Unis (pour Hawaii et les Samoa américaines) et la Nouvelle-Zélande " (p. 78). Cela permet de conserver un certain équilibre entre les (petits) membres, mais les prive également de leur soutien financier. Selon Sémir Al Wardi, il ne faudrait pourtant pas voir l'émergence récente de ces groupes comme une volonté de rivaliser avec les groupes mélanésien et micronésien. D'autant plus qu'ils sont tous concernés par l'instabilité politique. Par ailleurs, il est certain pour l'auteur que les indépendantistes polynésiens utiliseront le GLP pour porter leurs revendications, comme l'avait fait le FLNKS avec le GFLM. Néanmoins, la Polynésie française peut s'exprimer dans ces groupes, dès lors qu'il ne sagit pas de relations internationales classiques dans un cadre étatique. Si c'était le cas, l'autorisation de la France serait nécessaire. Pourtant, l'action concertée des deux groupes polynésiens au sein du forum a abouti, à la demande des Samoa, Nauru, Tuvalu, Salomon et du Timor oriental, à ce que l'oNu accepte la réinscription du territoire français le 17 mai 2013.

Confrontée à l'impératif croissant des transformations environnementales (changement et réchauffement climatiques, pollutions, énergies...), la région océanienne aborde ces problématiques avec intérêt. Ainsi, les territoires français du Pacifique Sud sont impactés par ces mutations environnementales. À partir de l'exemple de la Polynésie française, l'article de Rudy Bessard (pp. 89-104) interroge les formes de leadership institutionnel et politique dans leurs rapports aux transformations environne- 
mentales. L'État et les principaux leaderships politiques polynésiens mobilisent des ressources politiques (institutionnelles, identitaires et rentières), qui se trouvent parfois en décalage avec le renouvellement des enjeux politiques et la mise à l'agenda de la question écologique. L'auteur souligne la nécessité, pour l'État et ses territoires, de saisir les enjeux politiques des transformations environnementales au XXI ${ }^{e}$ siècle, afin de coordonner de nouvelles formes de réponses publiques.

En conclusion de ce dossier, son Excellence l'Ambassadeur de France dans le Pacifique, Christian Lechervy, propose une vision globale et une conceptualisation de la politique régionale de la France dans le Pacifique Sud (pp. 105120). La "politique Pacifique» de la France prend forme à partir de plusieurs stratégies sous-régionales reposant sur la recherche d'une plus grande efficacité diplomatique de l'État en Asie-Pacifique. Dans son "nouveau projet de puissance ", la France s'appuie sur une stratégie d'insertion multilatérale prenant à la fois en compte les espaces Asie-Pacifique, Pacifique, mélanésien et polynésien. Cette approche plurielle des vecteurs d'intégration régionale illustre ainsi un renouvellement de la réflexion étatique sur la région océanienne à partir des collectivités du Pacifique.

L'intégration régionale représente alors un levier pertinent pour favoriser la compréhension et la coopération des territoires français du Pacifique face aux dynamiques universelles relatives aux transformations mondialisées en termes géopolitiques, environnementaux et technologiques. Il ressort de ces différentes approches que le processus d'intégration régionale autour des territoires français du Pacifique oscille d'un côté, entre une insertion régionale intra et extra-territoriale appuyée par la France et l'Union européenne dans une perspective géopolitique et stratégique, et, de l'autre, le développement d'une identité régionale au sein des institutions océaniennes, avec pour horizon un projet commun pan-océanien intégrant les territoires français du Pacifique à la " mer d'îles » océaniennes.

\section{BIBLIOGRAPHIE}

Agniel Guy, 2008. La gestion de l'environnement dans le Pacifique. Moyens et objectifs. Journal de la Société des Océanistes 126-127: Spécial environnement dans le Pacifique, Jean Trichet et Isabelle Leblic (éds), pp. 145-151 (http://jso.revues.org/3842).

Al wardi Sémir et Jean-Marc Regnault, 2011. Tahiti en crise durable. Un lourd héritage, $\mathrm{Pa}$ peete, Éd. de Tahiti, 127 p.
Angleviel Frédéric, 2006. La coutume et l'État à Wallis-et-Futuna : un mariage de raison, in P. De Deckker (éd.), Figures de l'État dans le Pacifique, Paris, L'Harmattan, pp. 49-66.

Argounès Fabrice, Sarah Mohamed-GaILLARD et Luc VACHer, 2011. Atlas de l'Océanie. Continent d'ìles, laboratoire du futur, Paris, Éditions Autrement, 80 p.

Babadzan Alain, 2009. Le spectacle de la culture. Globalisation et traditionalismes en Océanie, Paris, L'Harmattan, 290 p.

Babadzan Alain (éd.), 1999. Journal de la Société des Océanistes 109 : Les politiques de la tradition. Identités culturelles et identités nationales dans le Pacifique, 140 p. (http://jso.revues. org/persee-246572).

BACH Daniel C. (éd.), 1998. Régionalisation, mondialisation et fragmentation en Afrique subsaharienne, Paris, Karthala, 319 p.

Barbe Dominique et Renaud Meltz (éds), 2013. Hermès 65 : Le monde Pacifique dans la mondialisation, CNRS Éditions, 210 p.

BaubÉrot Jean et Jean-Marc Regnault (éds), 2008. Les relations Églises/autorités outre-mer, de 1945 à nos jours, Paris, Les Indes savantes, 219 p.

BAYART Jean-François, 1999. L'Afrique dans le monde : une histoire d'extraversion, Critique internationale 5-5, pp. 97-120.

Bessard Rudy, 2013. Pouvoir personnel et ressources politiques. Gaston Flosse en Polynésie française, thèse de science politique dirigée par Daniel bourmaud, université de Pau, $810 \mathrm{p}$.

Budoc Rémy Louis, 2012. Pour un renforcement de la coopération régionale des Outremer, Conseil économique social et environnemental, JORF.

Cerf Patrick, 2007. La domination des femmes à Tahiti. Des violences envers les femmes au discours du matriarcat, Papeete, Au Vent des îles, 521 p.

Chor Yoonah, 2010. L'importance de l'Union européenne dans les territoires français du Pacifique, in M. Chatti et al., Pouvoir(s) et politique(s) en Océanie, actes du XIX ${ }^{e}$ colloque CORAIL, Paris, L'Harmattan, pp. 151-163.

Crocombe Ron, 2007. Asia in the Pacific Islands: Replacing the West, Suva, University of South Pacific.

Dabene Olivier, 2009 (6 juin). Intégrations régionales, Observatoire politique de l'Amérique latine et des Caraïbes (opALC) (http:// www.sciencespo.fr). 
DAvid Gilbert, 2008. La pêche côtière océanienne et son environnement. La dialectique instabilité/stabilité au fil de l'eau, Journal de la Société des Océanistes 126-127: Spécial environnement dans le Pacifique, Jean Trichet et Isabelle Leblic (éds), pp. 247-270 (http://jso. revues.org/4352).

—, 2011. Le Vanuatu côté océan : La révolution bleue et les premières années de l'indépendance, Journal de la Société des Océanistes 133, pp. 353-366 (http://jso.revues.org/6540).

De Deckker Paul, 2002. Le Pacifique: à la recherche du développement dans un espace émietté, Les outre-mers face à l'intégration européenne et à la mondialisation, Revue française d'administration publique (ENA) 101, pp. 157 168 (http://www.cairn.info/revue-francaise-dadministration-publique-2002-1.htm).

Dobell Graeme, 2013. France in the Pacific: ambiguity and ambition, The Strategist (http:// www.aspistrategist.org.au/france-in-the-pacific-ambiguity-and-ambition/).

Dousset Laurent, Barbara GlowCZewski et Marie Salaün (éds), 2014. Les sciences humaines et sociales dans le Pacifique Sud. Terrains, questions et méthodes, Marseille, Pacific Credo Publications, Cahiers du Credo, 400 p.

Faberon Jean-Yves, Viviane Fayaud et Jean-Marc Regnault (éds), 2011. Destins des collectivités politiques d'Océanie. Peuples, populations, nations, Etats, territoires, pays, patries, communautés, frontières, Marseille, Presses de l'université Aix-Marseille (PUAM), 2 vol., 878 p.

Faberon Jean-Yves, 2008. L'idée fédérale en Nouvelle-Calédonie depuis les accords de Matignon, in J.-M. Regnault et V. Fayaud (éds), La Nouvelle-Calédonie, vingt années de concorde, 1988-2008, Paris, Publications de la Société française d'histoire d'Outre-Mer, pp. 111-119.

Faberon Kareen, 2003. Partager la NouvelleCalédonie : l'invention de la régionalisation, in J.-M. Regnault (éd.), François Mitterrand et les territoires français du Pacifique (19811988): mutations, drames et recompositions; enjeux internationaux et franco-français, Paris, Les Indes Savantes, pp. 321-333.

FABry Véronique, 2005. L'Outre-mer dans les ensembles régionaux, Pouvoirs 113, pp. 159-170.

Fer Yannick (éd.), 2014. Diasporas asiatiques dans le Pacifique. Histoire des représentations et enjeux contemporains, Paris, Les Indes Savantes, $198 \mathrm{p}$.

Fisher Denise, 2012. France: « in " or " of » the South Pacific region?, Journal de la Société des Océanistes 135, pp. 185-200 (http://jso. revues.org/6702).

-, 2013. France in the South Pacific: Power and Politics, Canberra, ANU e-press.

FRY Greg, 2005. 'Pooled Regional Governance' in the Island Pacific: Lessons from History, in S. Chand (ed.), Pacific Islands Regional Integration and Governance, Canberra, Asia-Pacific Press, pp. 89-104.

GAY Jean-Christophe, 2008. L'outre-mer français : Un espace singulier, Paris, Belin, $232 \mathrm{p}$.

Giesen Klaus-Gerd, 2011. Les États insulaires d'Océanie dans l'économie politique internationale, in J.-Y. Faberon et al., Destins des collectivités politiques d'Océanie. Peuples, populations, nations, Etats, territoires, pays, patries, communautés, frontières, Marseille, Presses de l'université Aix-Marseille (PUAM), pp. 521-530.

GrafF Stéphanie, 2012. Quand combat et revendication kanak ou politique de l'État français manient indépendance, décolonisation, autodétermination et autochtonie en Nouvelle-Calédonie, Journal de la Société des Océanistes 134, pp. 61-83 (http://jso.revues. org/6647).

Grant Andrew et Fredrik Söderbaum, 2003. The New Regionalism in Afric, Aldershot Ashgate, $243 \mathrm{p}$.

Guiot Hélène et Isabelle Leblic (éds), 2006. Journal de la Société des Océanistes 122-123: Spécial Wallis-et-Futuna, 186 p. (http://jso. revues.org/507).

Hau'ofa Epeli, 2013 (éd. or. 1993). Notre mer d'îles, Arue (Tahiti), Pacific Islanders éd., 56 p. (trad. de Our Sea of islands, in E. Waddel, V. Naidu and E. Hau'ofa (eds), A New Oceania: rediscovering our sea of islands, School of Social and Economic Development, University of the South Pacific, Suva, pp. 2-16).

—, 2015 (éd. or. 1997). The Ocean in Us, in Dreadlocks in Oceania, vol. 1, pp. 124-148], L'océan est en nous, Arue (Tahiti), Pacific Islanders ed., 56 p.

—, 2015 Un passé à recomposer, Arue (Tahiti), Pacific Islanders Ed., 56 p. (trad. de Pasts to Remember, in Remembrance of Pacific pasts. Honolulu, University of Hawai'i Press. Ed. Robert Borowsky, pp. 453-471).

Henningham Stephen, 1991. France in the South Pacific in the 1980's: An Australian perspective, JSO 91-92, pp. 21-45.

Hettne Björn and Fredrik Söderbaum, 2000. Theorizing the Rise of Regionness, New Political Economy 5-3, pp. 457-473. 
Hugon Philippe, 2003. Les théories de la régionalisation, in $\mathrm{P}$. Hugon (éd.), Les économies en développement à l'heure de la régionalisation, Paris, Karthala, pp. 33-80.

INSTITUT DE LA STATISTIQUE ET DES ÉTUDES ÉCONOMiQues de Nouvelle-CalÉdonie (ISEe), http://www.isee.nc/.

Institut de LA STATISTique de Polynésie FRANÇAISE (ISPF), http://www.ispf.pf/.

Kochenov Dimitry (ed.), 2011. The EU Law of the Overseas: Outermost regions, Associated Overseas Countries and Territories, Territories Sui Generis, The Netherlands, Kluwer Law International BV.

Kotra Walles, 2009. Conversations calédoniennes. Rencontre avec Jacques Lafleur, Lieu, Au Vent des îles, 130 p.

LABORIE Léonard, 2013. "Via Pacific ». Télégraphie, impérialismes et mondialisation dans le Pacifique au XIXe siècle, in D. BARBE et R. MELTZ (éds), Hermès 65: Le monde Pacifique dans la mondialisation, CNRS Éditions, pp. 132-135.

Lal Brij V., 2007. Pacific history matters, Journal de la Société des Océanistes 125, pp. 193200 (http://jso.revues.org/894).

Leblic Isabelle, 1993. Les Kanak face au développement. La voie étroite, Grenoble, Presses universitaires de Grenoble.

Leblic Isabelle (éd.), 2003. Journal de la Société des Océanistes 117 : Nouvelle-Calédonie, 150 ans après la prise de possession, $178 \mathrm{p}$. (http:// jso.revues.org/1242).

Mara Ratu Sir Kamisese, 1997. The Pacific Way: A Memoir, Honolulu, University of Hawai'i Press.

Massau Serge, 2011. Paroles d'un autochtone. Entretiens avec le sénateur Richard Arïihau Tuheiava, Papeete (Tahiti), Haere Po, 161 p.

Merceron François et Jean Morschel, 2013. Tahiti et ses périphéries insulaires : formation et crise d'un espace centralisé, Hermès 65, CNRs Éditions, pp. 56-66.

Meyer Angela, 2006. Lintégration régionale et son influence sur la sécurité et la stabilité d'États faibles. L'exemple de quatre États centrafricains, thèse de Science politique dirigée par Bertrand Badie et Ingfrid Schüntz-Müller, IEP-Paris.

Mohamed-gaillard Sarah, 2010. L'archipel de la puissance? La politique de la France dans le Pacifique Sud de 1946 à 1998, Bruxelles, Berlin, New York, Éditions PIE Peter Lang, 428 p.
-, 2012. Les enjeux internationaux en Océanie: contexte historique et perspectives historiographiques, Journal de la Société des Océanistes 135: Relations internationales et régionales, Sarah Mohamed-Gaillard (éd.), pp. 177-184 (http://jso.revues.org/6701).

Mohamed-gaillard Sarah (éd.), 2012. Journal de la Société des Océanistes 135 : Relations internationales et régionales, 54 p. (http://jso. revues.org/6687).

Mokzadem Hamid, 2010. Le destin commun à l'épreuve du corps électoral en NouvelleCalédonie, in M. Chatti et al., Pouvoir(s) et politique(s) en Océanie, Actes du XIx ${ }^{e}$ colloque CORAIL, Paris, L'Harmattan, pp. 91-132.

Monnerie Denis, 2014. Les échanges en Océanie et l'anthropologie, in Laurent Dousset et al. (éds), Les sciences humaines et sociales dans le Pacifique Sud. Terrains, questions et méthodes, Marseille, Pacific Credo Publications, Cahiers du CREDO, pp. 127-159.

Mrgudovic Nathalie, 2003. Nouvelle-Calédonie ou Kanaky : perceptions régionales du "caillou ", Journal de la Société des Océanistes 117 : Nouvelle-Calédonie, 150 ans après la prise de possession, Isabelle Leblic (éd.), pp. 281297 (http://jso.revues.org/1323).

-, 2008. La France et le Pacifique Sud. Les enjeux de la puissance, Paris, L'Harmattan, 440 p.

Overton John, Gerard Prinsen, Warwick E. MurraY et Nicki Wrighton, 2012. Reversing the Tide of Aid: Investigating Development Policy Sovereignty in the Pacific, Journal de la Société des Océanistes 135, pp. 229-242 (http://jso.revues.org/6713).

Panoff Michel, 1989. Tahiti métisse, Paris, Denoël, $296 \mathrm{p}$.

Polanyi Karl, 2009 (1 $1^{\mathrm{e}}$ éd. 1944). La grande transformation: Aux origines politiques et économiques de notre temps, Paris, Gallimard, $476 \mathrm{p}$.

RALLU Jean-Louis et Gérard BAUdChON, 2003. Dynamiques socio-démographiques des populations des том des années 1980, in J.-M. Regnault (éd.), François Mitterrand et les territoires français du Pacifique..., Paris, Les Indes savantes, pp. 407-415.

Regnault Jean-Marc (éd.), 2003. François Mitterrand et les territoires français du Pacifique (1981-1988). Mutations, drames et recompositions. Enjeux internationaux et franco-français, Paris, Les Indes savantes, 583 p.

Regnault Jean-Marc, 2013. La France, l'ONU et les décolonisations tardives. L'exemple des terres françaises d'Océanie, Marseille, PUAM, 250 p. 
Rocard Michel, Une politique française du Pacifique ?, colloque du 17 janvier 2013, Sénat, rapport d'information $n^{\circ} 293$, délégation sénatoriale à l'outre-mer (http://www.senat.fr/ rap/r12-293/r12-2933.html).

Ro'i Laïsa, 2013. L'intégration régionale océanienne. Enjeux, contraintes et perspectives, thèse de Sciences économiques dirigée par M. A. Sénégas et J. Le Gallo, université de Bordeaux 4 (http://www.theses. fr/2013BOR40025/document).

SAND Christophe, 2010. Lapita calédonien. Archéologie d'un premier peuplement insulaire océanien, Paris, Société des Océanistes, Travaux et documents océanistes 2 .

Saura Bruno, 2008. Tahiti Ma'ohi. Culture, identité, religion et nationalisme en Polynésie française, Papeete, Au Vent des îles, 529 p.

Service territorial de la STATISTIQUe ET DES ÉTUdes ÉCONOMIQUeS DE WALLIS-ET-FutUNA (STSEE), http://www.spc.int/prism/wf/.

Soriano Éric, 2014. La fin des Indigènes en Nouvelle-Calédonie. Le colonial à l'épreuve du politique (1946-1976), Paris, Karthala, 312 p.

Smouts Marie-Claude (éd.), 2007. La situation postcoloniale: les postcolonial studies dans le débat français, Paris, Presses de la Fondation nationale des Sciences politiques, $451 \mathrm{p}$.
Soule Marc, 2003. Wallis-et-Futuna (19811995) : entre identité coutumière et volonté de désenclavement, in J.-M. Regnault (éd.), François Mitterrand et les territoires français $d u$ Pacifique..., Paris, Les Indes savantes, pp. 435-440.

Tcherkezoff Serge, 2009. Polynésie/Mélanésie. Linvention française des "races" et des régions de l'Océanie, Papeete, Au Vent des îles.

Trichet Jean et Isabelle Leblic (éds), 2008. Journal de la Société des Océanistes 126-127: L'environnement dans le Pacifique, 166 p. (http://jso.revues.org/1804).

Van Langenhove Luk, 2003. Theorizing regionhood, UNU/CRIs e-Working Papers, 38 p. (http://www.cris.unu.edu/fileadmin/workingpapers/paper\%20regionhood.pdf).

Vernaudon Jacques, 2010. Les langues océaniennes des collectivités françaises du Pacifique. Enjeux politiques, enjeux pédagogiques, in M. Chatti et al., Pouvoir(s) et politique(s) en Océanie, actes du XIx ${ }^{\mathrm{e}}$ colloque CORAIL, Paris, L'Harmattan, pp. 165-179.

Wittersheim Éric, 2014. Noir c'est noir : l'«Africanisation " du Pacifique en question, in L. Dousset et al. (éds), Les sciences humaines et sociales dans le Pacifique Sud. Terrains, questions et méthodes, Marseille, Pacific CREDo Publications, Cahiers du CREDO, pp. 377-400.

NB. Cette introduction est disponible en anglais sur jso.revues.org / This presentation, « Regional Perspectives and Oceanian Variations for the French Territories», is avalaibale online in English, see jso.revues.org 